\title{
THE PARKES MULTIBEAM PULSAR SURVEY: PRELIMINARY RESULTS
}

\author{
N. D'Amico ${ }^{1}$, A.G. Lyne ${ }^{2}$, R.N. Manchester ${ }^{3}$, F.M. Camilo², V.M. Kaspi ${ }^{4}$, \\ J. Bell ${ }^{3}$, I.H. Stairs ${ }^{2}$, F. Crawford ${ }^{4}$, D. Morris ${ }^{2}$, A. Possenti ${ }^{1}$ \\ 1) Osservatorio Astronomico di Bologna, via Ranzani 1, 40127 Bologna, \\ and Istituto di Radioastronomia del CNR, via Gobetti 101, 40129 Bologna, Italy \\ 2) University of Manchester, Jodrell Bank Observatory, \\ Macclesfield, Cheshire, SK11 9DL, UK \\ 3) Australia Telescope National Facility, CSIRO, PO Box 76, \\ Epping NSW, 1710, Australia \\ 4) Massachusetts Institute of Technology, Center for Space Research, 70 Vassar Street, \\ Cambridge, MA 02139, USA
}

\begin{abstract}
A high-frequency survey of the Galactic plane for radio pulsars is in progress, using the multibeam receiver on the 64-m Parkes radiotelescope. We describe the survey motivations, the observing plan and the inital results. The survey is discovering many pulsars, more than 500 so far. Eight of the new pulsars are binary, one with a massive companion. At least eight are young, with characteristic ages of less than $100 \mathrm{kyr}$. Two of these (Kaspi et al, this Conference) have surface dipole magnetic field strengths greater than any other known radio pulsar.
\end{abstract}

KEYWORDS: methods: observational; pulsars: general; pulsars: searches.

\section{INTRODUCTION}

Young pulsars are relatively rare objects in the pulsar population because they evolve rapidly, so on average their distance is relatively high. They are usually found at low Galactic latitudes, close to their places of birth, where their detection is limited by the high background temperature and by the broadening of pulses due to dispersion and interstellar scattering. On the other hand, they are interesting objects for many reasons: they are likely to be $\gamma$-ray sources; they exibhit rotational glitches, which are of interest in the understanding of the interior structure of neutron stars; they are likely to be associated with supernova remnants.

High frequency $(\nu \simeq 1400 \mathrm{MHz}$ ) surveys (Clifton et al, 1992; Johnston et al 1992) and searches (Kaspi et al, 1996; Manchester, D'Amico \& Tuohy, 1985) for young, low latitude distant pulsars proved to be successful, because the contribution of the Galactic synchrotron radiation to the radiotelescope system temperature is highly reduced, because the effect of dispersion is more easlily removed, and 


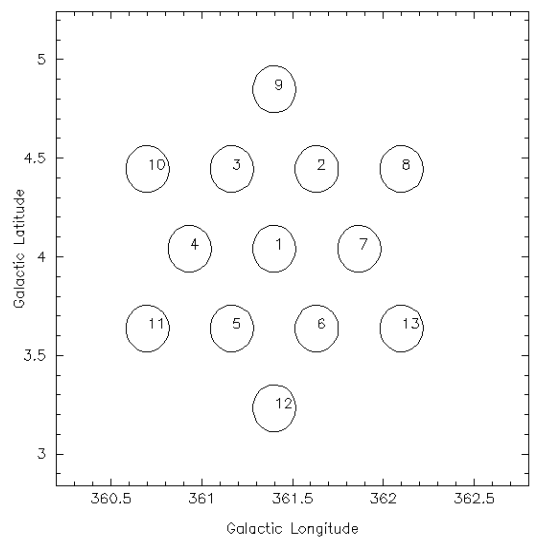

FIGURE 1. Beam pattern of the multibeam receiver at Parkes.

because the broadening of pulses due to interstellar scattering varies with frequency aproximately as $\nu^{-4.4}$. Triggered by the above motivations, we are undertaking a new survey for pulsars along the Galactic plane at 1.4 Ghz, using the 13-element multibeam receiver recently installed on the 64-m Parkes radiotelescope. In this paper we present the experiment configuration, the survey plan and the preliminary results of about $50 \%$ of the survey.

\section{THE MULTIBEAM SURVEY}

Each beam of the multibeam receiver system at Parkes is approximately $0.23^{\circ}$ wide and the beams centres are spaced 2 beamwidth apart (see Fig. 1). The survey pointings are interleaved to give complete sky coverage on a hexagonal grid containing a total of 2670 pointings of 13 beams each. The parameters of the present experiment and those of two previous high frequency surveys of the Galactic plane are summarized in Table 1. Thanks to the long integration time adopted (35-min) and the high sensitivity of the new receiver system, the present survey has a sensitivity 7 times better than previous surveys. Fig 2 . shows the theoretical sensitivity as a function of the pulsar period and dispersion measure.

\section{RESULTS AND DISCUSSION}

So far we have observed about 1600 pointings, $90 \%$ of which are analysed, corresponding to about $50 \%$ of the total survey region. The data reduction system is similar to that used in the Parkes low frequency survey (Manchester et al, 1996), and is carried out on a network of workstations. Because of the relatively long integrations adopted, we complement the standard search analysis with "acceleration search" to take into account possible binary motions. To date we have discovered 
TABLE 1 . Three $20 \mathrm{~cm}$ pulsar surveys

\begin{tabular}{llll}
\hline & Jodrell Bank & Parkes & Parkes \\
\hline Latitude range, $|b| \ldots \ldots$ & $<1^{\circ}$ & $<4^{\circ}$ & $<5^{\circ}$ \\
Longitude range, $l \ldots \ldots$ & $-5^{\circ} \ldots 100^{\circ}$ & $-90^{\circ} \ldots 20^{\circ}$ & $-100^{\circ} \ldots 50^{\circ}$ \\
Center frequency $(\mathrm{MHz})$ & 1400 & 1520 & 1374 \\
Number of beams $\ldots \ldots$ & 1 & 1 & 13 \\
Integration time $(\mathrm{min})$. & 10 & 2.5 & 35 \\
Sample interval $(\mathrm{ms}) \ldots$ & 2.0 & 1.2 & 0.25 \\
Bandwidth $(\mathrm{MHz}) \ldots \ldots$ & $2 \times 8 \times 5$ & $2 \times 64 \times 5$ & $2 \times 96 \times 3$ \\
$S_{\text {sys }}(\mathrm{Jy}) \ldots \ldots \ldots \ldots \ldots$ & 60 & 70 & 36 \\
$S_{\text {min }}(\mathrm{mJy}) \ldots \ldots \ldots$. & 1.2 & 1.0 & 0.15 \\
Pulsars found/detected. & $40 / 61$ & $46 / 100$ & $513+/ 703+$ \\
Reference ............ & Clifton et al. & Johnston et al. & this work \\
\hline \hline
\end{tabular}

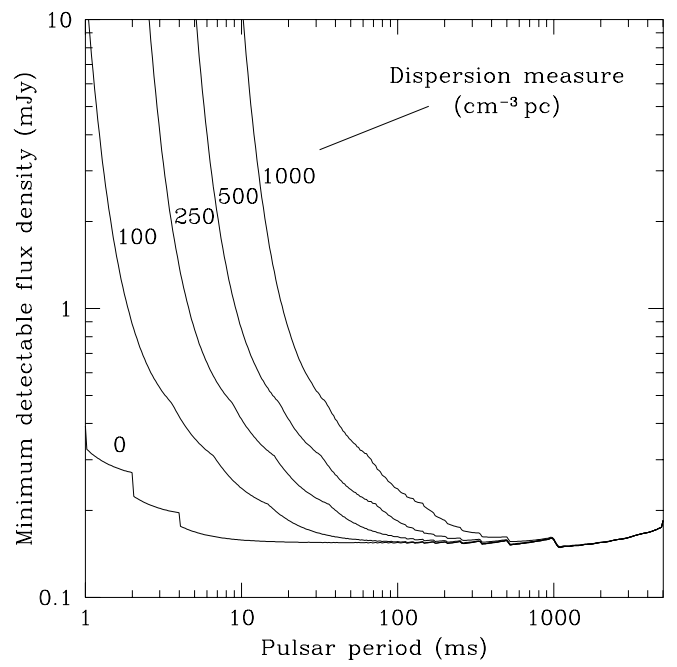

FIGURE 2. Theoretical sensitivity of the Parkes multibeam pulsar survey as a function of period and DM for a pulsar on the centre beam. 
TABLE 2. New binary pulsars

\begin{tabular}{lcccccc}
\hline PSR J & $\begin{array}{c}P \\
(\mathrm{~ms})\end{array}$ & $\begin{array}{c}\tau_{c} \\
\left(10^{6} \mathrm{y}\right)\end{array}$ & $\begin{array}{c}\text { Distance } \\
(\mathrm{kpc})\end{array}$ & $\begin{array}{c}P_{b} \\
(\mathrm{~d})\end{array}$ & Ecc. & $\begin{array}{c}\text { Min. } M_{c} \\
\left(M_{\odot}\right)\end{array}$ \\
\hline $\mathrm{J} 1232-6501$ & 88.28 & 1400 & 10.0 & 1.863 & 0.00 & 0.15 \\
$\mathrm{~J} 1904+04$ & 71.09 & - & 4.0 & 15.750 & 0.04 & 0.23 \\
$\mathrm{~J} 1810-2005$ & 32.82 & 4000 & 4.0 & 15.012 & 0.00 & 0.29 \\
$\mathrm{~J} 1453-58$ & 45.25 & - & 3.3 & 12.422 & 0.00 & 0.88 \\
$\mathrm{~J} 1435-60$ & 9.35 & - & 3.2 & 1.355 & 0.00 & 0.90 \\
& & & & & & \\
$\mathrm{~J} 1811-1736$ & 104.18 & 950 & 5.9 & 18.779 & 0.83 & 0.87 \\
$\mathrm{~J} 1141-6545$ & 393.90 & 1.45 & 3.2 & 0.198 & 0.17 & 1.01 \\
& & & & & & \\
$\mathrm{~J} 1740-3052$ & 570.31 & 0.36 & 10.8 & 231.039 & 0.58 & 11.07 \\
\hline
\end{tabular}

513 new pulsars, and have detected 190 known pulsars. Accounting for the fact that so far we searched the regions closest to the Galactic plane, we believe that the number of new discoveries for the entire survey should be somewhat over 800 .

Timing observations of the newly discovered pulsars are carried out at Jodrell Bank and Parkes. Observations are made at intervals of $4-8$ weeks, or more closely spaced when pulse-counting statistics need to be resolved. Full timing solutions have been obtained so far for 80 pulsars. At least eight of the new discoveries are young pulsars $\left(\tau_{c}<10^{5}\right.$ years $)$. Two radio pulsars with the highest known surface magnetic field have been discovered (Kaspi et al, these proceedings). One of these objects, PSR J1119-6127 is very young, with as characteristic age, $\tau_{c}=1600$ years. For this pulsar we also measured a braking index $n=3.0 \pm 0.1$.

So far, eight of the newly discovered pulsars proved to be members of binary systems, including a pulsar (PSR J1811-1736) in a highly eccentric binary system (Lyne et al 1999) and a pulsar (PSR J1740-3052) with a very massive companion $\left(\simeq 11 \mathrm{M}_{\odot}\right)$. The basic parameters of the binary pulsars are shown in Table 2 .

\section{REFERENCES}

Clifton, T.R., Lyne, A.G., Jones, A.W., McKenna, J., Ashworth, M. 1992, MNRAS, 254, 177

Johnston, S., Lyne, A.G., Manchester, R.N., Kniffen, D.A, D'Amico, N., Lim, J., Ashworth, M. 1992, MNRAS, 255, 401

Kaspi, V.M., Manchester, R.N., Johnston, S., Lyne, A.G., D'Amico, N. 1996, AJ, 111, 2028

Lyne, A.G. et al 1999, MNRAS, in press; astro-ph/9911313

Manchester R.N., D'Amico, N., Tuohy, I.R. 1985, MNRAS, 212, 975

Manchester R.N., et al 1996, MNRAS, 279, 1235 